\title{
EUV Studies of Solar System Objects: A Status Report
}

\section{SUPRIYA CHAKRABARTI ${ }^{1}$ AND G. RANDALL GLADSTONE ${ }^{2}$}

\author{
${ }^{1}$ Center for Space Physics, Boston University, \\ 725 Commonwealth Avenue, Boston, MA 02215, USA \\ ${ }^{2}$ Southwest Research Institute, PO Drawer 28510, San Antonio, TX 78228, USA
}

\begin{abstract}
EUV studies have contributed substantially to our understanding of the physical and chemical properties the Sun, planets, and their satellites. Although the spectroscopic data set is limited to Venera 11/12, Voyager 1/2, Astro 1/2, EUVE, Galileo, and a handful of sounding rocket experiments, these data have provided important insights regarding the atmospheres and surfaces of several planets and satellites to the point where rudimentary comparative planetology can be conducted. In this paper we highlight some of these results.
\end{abstract}

\section{Introduction}

The EUV spectral region is rich in emission features from the most common planetary atmospheric species. Recently, it has also been proposed to be useful for studies of the surfaces of solar system bodies without atmospheres. Spectrographs aboard planetary spacecraft, most notably the Voyagers, have established the general EUV/FUV spectral characteristics of the upper atmospheres and plasma environments of most of the planets. The EUVE mission continues to provide new insights into solar system objects. The space-shuttle-based Astro missions and sounding rocket experiments have also added to our understanding of the solar system through their EUV observations.

The richness of the EUV spectral region for planetary studies has been discussed by Feldman \& Bagenal (1991) (see their Table 1). Extreme ultraviolet emission features include transitions of atoms and their ions (e.g., $\mathrm{H}, \mathrm{He}, \mathrm{He}^{+}, \mathrm{Ne}, \mathrm{Ar}, \mathrm{O}, \mathrm{O}^{+}, \mathrm{O}^{++}, \mathrm{N}$, $\mathrm{N}^{+}, \mathrm{N}^{++}, \mathrm{S}^{+}, \mathrm{S}^{++}, \mathrm{S}^{+++}$) as well as molecules (e.g., $\mathrm{H}_{2}$ and $\mathrm{N}_{2}$ ). Through occultation studies, one can also infer the altitude distribution of abundant EUV-absorbing molecules (e.g., $\mathrm{O}_{2}$ and $\mathrm{N}_{2}$ on Earth, or hydrocarbons on the giant planets).

In this paper we provide a summary of EUV observations of solar system objects. The discussions will be broken into observations of the study of the Moon, the terrestrial planets, the jovian planets, the interplanetary medium, and comets.

\section{The Moon}

There have been only a small number of studies on EUV emissions from the Moon. An EUV spectrophotometer on the Mariner 10 spacecraft observed the Moon in the $550-1250 \AA$ range and obtained a geometric albedo of $2-10 \%$ at a solar phase angle of $74^{\circ}$ (Wu \& Broadfoot 1977).

Prompted by the ROSAT soft X-Ray observation of the Moon (Schmitt et al. 1991), Edwards et al. (1991) simulated lunar EUV emissions from L- and M-shell X-Ray fluorescence. The authors predicted much higher emission peaks in the $90-500 \AA$ range from this process than from the reflected solar spectrum.

During the all-sky survey phase of the EUVE operations, EUVE scanned the Moon on several occasions. Gladstone et al. (1994) used this data to estimate the solar EUV 


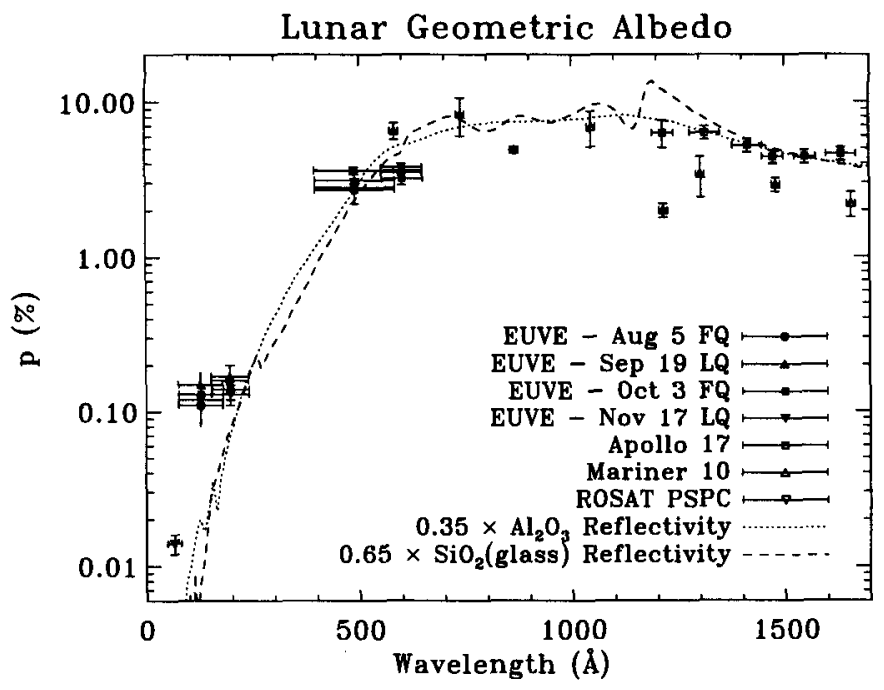

FIGURE 1. EUV albedo of lunar surface obtained by EUVE and other experiments.

irradiance and its variation. The authors used the bulk reflectivities of $\mathrm{SiO}_{2}$ and $\mathrm{Al}_{2} \mathrm{O}_{3}$ (Phillip 1985 and Gervais 1991) to explain the photometric observations (Figure 1). The average geometric albedos obtained in the $150-650 \AA$ range varied from $0.15 \%$ to $3.5 \%$ with an upper limit of $0.13 \%$ in the $75-180 \AA$ range. The authors found that the primary features are consistent with reflected sunlight, rather than the X-ray fluorescence emissions suggested by Edwards et al. (1991). Subsequently, Gladstone (1994) used the $E U V E$ spectrometer to obtain the EUV spectrum of the Moon, which indicated that the observed signal is primarily due to reflected sunlight.

\section{Terrestrial Planets}

Although there have been numerous EUV observations of Earth's upper atmosphere, only a single EUV observation of Mars and a handful for Venus have been reported (e.g., Krasnopolsky et al. 1994; Bertaux et al. 1981; Hord et al. 1991). As a result, EUV observations of terrestrial planets remain an almost unexplored domain. Even the first round of discovery missions will not fill this gap.

Terrestrial EUV observations started with broadband photometric measurements from sounding rockets and satellites which obtained the intensity distributions of the bright features from the geocorona (H I Lyman $\alpha$ and He I $584 \AA$ ), nighttime ionosphere (O I $911 \AA, 1304 \AA$, and $1356 \AA$ features), plasmasphere (He II $304 \AA$ ), and the interplanetary medium (He I $584 \AA$ and He II $304 \AA$ ) [for an excellent review of Earth's EUV airglow see Meier (1991)]. Detailed experimental and modeling studies have shown evidence for the upflow of ionospheric ions in the magnetosphere (Chiu et al. 1986), detected the presence of a non-thermal population of atomic oxygen (Cotton et al. 1993), inferred solar line center flux and line width from He I $584 \AA$ airglow (Bush \& Chakrabarti 1995), and obtained the ionospheric temperature from the shape of $\mathrm{O} I$ recombination continuum (Feldman et al. 1992).

The atmospheres of Mars and Venus have been observed in the FUV from a number of U.S. and Soviet spacecraft. He I $584 \AA$ emission from Venus was observed by the Ven- 
Galileo Venus EUV Spectrum

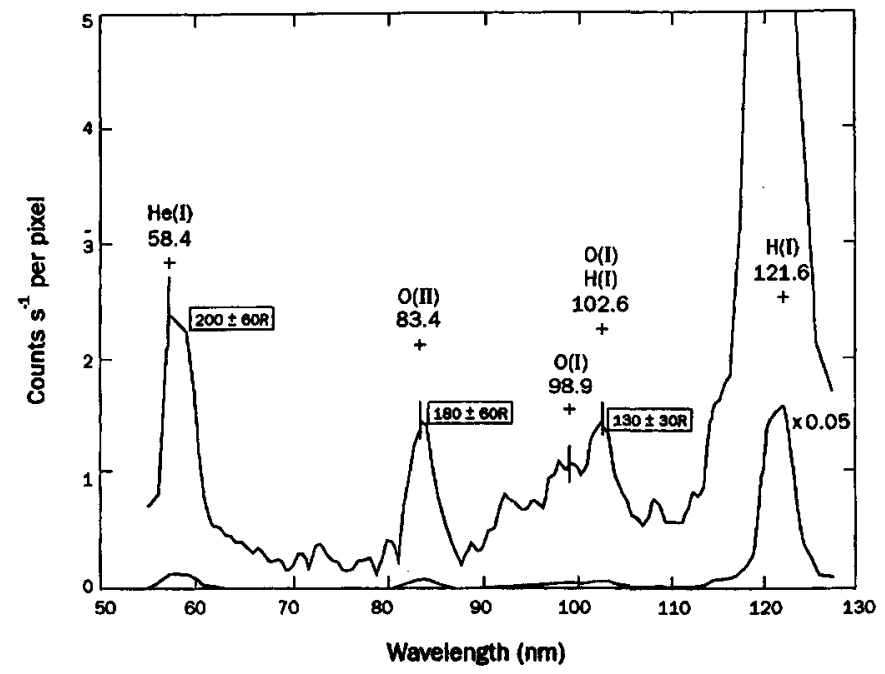

FIGURE 2. Venus airglow spectrum obtained by the Galileo UV spectrograph.

era 11/12 spacecrafts (Chassefiere et al. 1986). O II $834 \AA$ emissions and, surprisingly, argon emissions at $869 \AA$ and $1048 \AA$ were also recorded by Venera missions. These observations were conducted by broadband instruments, however, which makes unambiguous determination of atmospheric composition a difficult task (Bertaux et al. 1981). In particular, the argon detections are especially suspect. On its trip to Jupiter, the Galileo spacecraft obtained an EUV spectrum of Venus (Hord et al. 1991), which includes several features due to $\mathrm{O}, \mathrm{O}^{+}$, $\mathrm{He}$ and $\mathrm{H}$ (Figure 2).

EUV spectroscopy of Mars and Venus can provide important clues for understanding not only the present structure of their upper atmospheres, but also their outgassing history. The first step towards that goal was achieved by the EUVE mission in January 1993 , when it observed the He I $584 \AA$ feature on Mars (Krasnopolsky et al. 1994). The observed spectrum shows the presence of martian helium emissions above the geocoronal background. The measured brightness of the He I $584 \AA$ emission was 43 Rayleighs, which results in an inferred lifetime of $5 \times 10^{4}$ years for helium in the martian atmosphere.

A recent sounding rocket experiment by S. A. Stern and colleagues obtained an EUV spectrum of Venus, and the HUT experiment aboard the Astro-2 mission obtained EUV spectra of Venus and Mars. These results, although they have not yet appeared in the literature, will undoubtedly provide new insights and improve our understanding of the upper atmospheres of the terrestrial planets.

\section{Jovian Planets}

Of the Jovian planets, Jupiter remains the most extensively studied at EUV wavelengths. The UVS experiments on the Voyager 1 and 2 spacecraft have provided the primary EUV spectroscopic data set for the giant planets and their satellites. The EUV spectra of the Jupiter, Saturn, and Uranus, when properly scaled by their heliocentric distances, appear very similar (Yelle et al. 1987). They do differ, however, above $1100 \AA$, 
and the UVS data at these longer EUV wavelengths have been used to infer upper atmospheric composition.

The highest spectral resolution observations of Jupiter in the EUV were obtained by the HUT spectrograph aboard the Astro-1 (Feldman et al. 1993) and Astro-2 missions. These measurements clearly indicate the hydrogen Lyman series of lines as well as fluorescence emissions excited by solar Lyman $\beta$.

The HUT observations. at superior spectral resolution have provided important new insight into the controversy over $\mathrm{H}_{2}$ band airglow emissions seen on Jovian planets. Feldman et al. (1993) noted that an electron impact excitation source due to atmospheric dynamo, such as that proposed by Clarke et al. (1987), is required to explain the data. This source is needed in addition to solar fluorescence (Yelle et al. 1987), which accounts for up to $22 \%$, and photoelectrons (Waite et al. 1983).

Recently, the $E U V E$ mission observed the Jupiter and Io system in conjunction with the comet Shoemaker-Levy 9 impact. The data show an increase of Jupiter's He I $584 \AA$ brightness by about a factor of 1.5 during the comet impact over its pre-impact value. Gladstone et al. (1995) surmise that the most likely explanation for the increase is the rising of helium in the impact-generated plumes from the well-mixed lower atmosphere into the depleted upper atmosphere. The extra helium in the upper atmosphere is then able to scatter more sunlight at $584 \AA$. An investigation of the EUV light curves obtained by $E U V E$ shows the He I $584 \AA$ enhancement during individual comet fragment impacts for the larger fragments $(\mathrm{G}, \mathrm{H}$, and $\mathrm{K}$ ). The 1-2 hour delay of the brightening of the signal indicates the time required for substantial lateral expansion of the plumes as the impact sites rotate toward the direction of Earth.

Strong EUV emissions from the Io Plasma Torus (IPT) were discovered by the UVS instrument during the flyby of Voyager 1 (Broadfoot et al. 1979). The IPT is the only substantial emitter of EUV radiation (rather than simply being a reflector of sunlight) in the solar system. Sulfur and oxygen ions in a relatively dense plasma torus $\left(\sim 10^{3} \mathrm{~cm}^{-3}\right)$ surrounding the orbit of Io are excited by electron impact to radiate at several characteristic EUV wavelengths. Most of the energy for the torus comes from Jupiter's rotation. As neutral sulfur and oxygen atoms escape from Io through sputtering or other mechanisms, they are likely to become ionized and join the torus population. As they do so, they are swept up by Jupiter's corotating magnetic field which, since it moves much faster than Io's orbital velocity around Jupiter, gives the newly-formed torus sulfur and oxygen ions an energy boost of about $525 \mathrm{eV}$ and $262 \mathrm{eV}$, respectively.

High-resolution EUV spectra of the IPT were obtained by the Astro-1 and Astro-2 missions. These spectra are totally dominated by emissions from the oxygen and sulfur ions (Moos et al. 1991). The EUVE spectrographs have also been used to study the IPT, and they have the advantage of being able to map out the spatial distribution of several of the EUV emissions over the entire torus (Hall et al. 1994). In addition to a strong dusk/dawn brightness asymmetry, the EUVE IPT data of the O II $539 \AA$ feature also seem to indicate the presence of other torus-like features inside the orbit of Io (Figure 3 ). $E U V E$ spectrometer data of the Io Plasma Torus before, during, and after the SL-9 impacts show no significant change in their relative brightnesses (Hall et al. 1995).

For the other outer planets, the Voyager UVS (Broadfoot et al. 1977) made extensive use of solar and stellar occultations to obtain atmospheric composition and temperature structure. This is possible due to the fact that the opacity of the atmosphere in different EUV wavelength regions can differ significantly (Broadfoot et al. 1989). 
EUVE Jupiter System $0^{+}$Images

\author{
Relative \\ Brightness
}

April 1, 1993

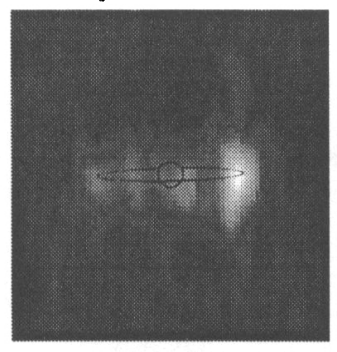

April 29, 1994

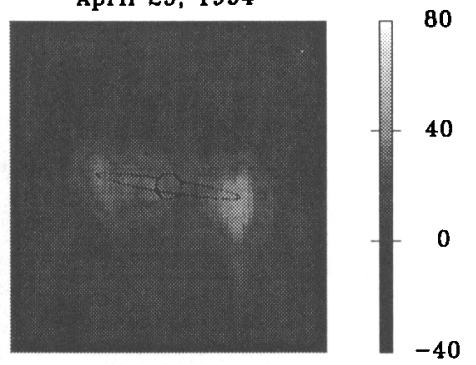

Figure 3. Images of the Jupiter (circle at the center) and Io Torus (dashed line) obtained at $539 \AA$ by EUVE. Note the presence of torus-like features inside Io's orbit.

\title{
5. Satellites
}

While passing Neptune, the Voyager 2 UVS experiment obtained an EUV spectrum of Triton (Broadfoot et al. 1989), which showed that the primary features are due to various band systems of $\mathrm{N}_{2}$. The other primary emission feature include the N II $1085 \AA$ and H I $1216 \AA$ lines. The authors noted that the data do not include positive detections of $\mathrm{CO}, \mathrm{Ar}$ and Ne. From the absolute brightness and day/night asymmetry of the observed intensity of $\mathrm{H}$ I Lyman $\alpha$, the authors concluded that photoelectrons and magnetospheric electrons might be responsible for some of the features.

The extended atmosphere of Triton, which resembles that of Pluto (and also somewhat that of Titan), also contributes to the formation of a proposed "plasma arc" (partial torus) around Neptune. Broadfoot et al. 1989 used this model to explain the presence of H I Lyman $\beta$ emissions in the aurora and the relative absence of H I Lyman $\alpha$.

\section{Comets}

A sounding rocket observation (Green et al. 1991) of Comet Austin in the $910-1180 \AA$ band at approximately $3 \AA$ resolution revealed an emission feature at $1128 \AA$ which the authors attributed to a forbidden line of atomic oxygen. Such a feature has not been observed in the laboratory or in the terrestrial atmosphere, nor in any other comet spectrum (Feldman et al. 1991), and has raised an interesting controversy regarding atomic spectroscopy [see, for example, Slanger (1991) and its response]. Green et al. 1991 argue that the optically thick conditions that might explain the presence of $1128 \AA$ emission might be present due to the large extent of the coma in a dynamically new comet.

The EUV data from this same sounding rocket flight were used to obtain limits on the relative abundances of cometary helium and argon (Stern et al. 1992). Although only upper limits to the abundances were possible, this was the first constraint of $\mathrm{He}$ and $\mathrm{Ar}$ abundances in comets. The authors found that the relative abundance of $\mathrm{He} / \mathrm{O}$ in comet Austin is $1.5 \times 10^{4}$ depleted and no more than 30 times enriched in Ar/O compared to solar abundances. The He/O depletion can be used to infer that the cometary nuclear ices are not always maintained within a few degrees of absolute zero. 


\section{The Interplanetary Medium}

There have been only limited observations of the interplanetary medium in the EUV. The Voyager UV spectrometer, with a $1.5 \times 10^{6}$ seconds exposure, has collected a spectrum of the sky in the direction of the North Galactic Pole (Holberg 1986). This observation, clearly contains the He I $584 \AA$ feature produced by resonance scattering of solar emissions by neutral helium atoms in the heliosphere. The interpretation of this emission has subsequently raised the possibility that the solar $584 \AA$ line is redshifted (Chassefiere et al. 1988).

The presence of 0.02 Rayleigh $\left(1.6 \times 10^{3}\right.$ photons $\left.\mathrm{cm}^{-2} \mathrm{sec}^{-1} \mathrm{ster}^{-1}\right)$ of He II $304 \AA$ emissions was observed in the photometric data obtained during the Apollo-Soyuz mission in the Earth's shadow cone (Paresce et al. 1981). These data were interpreted as due to multiple scattering of solar emissions, either by plasmaspheric $\mathrm{He}^{+}$ions or by $\mathrm{He}^{+}$in the interstellar medium produced by photoionization of He. A more recent analysis of $E U V E$ survey observations obtained brightnesses of 1.30 Rayleigh, 0.040 Rayleigh, and 0.029 Rayleigh for the He I $584 \AA, 537 \AA$, and the He II $304 \AA$ emissions, during an exposure of 575,232 seconds looking down Earth's shadow (Jelinsky et al. 1995). As with the Apollo-Soyuz data, it is unclear what portion of these signals is due to multiple scattering in Earth's geocorona and plasmasphere.

On its way to Jupiter the Galileo spacecraft has made routine spectroscopic observations of the interplanetary medium (Hord et al. 1991). Although only the hydrogen Lyman $\alpha$ observations have been analyzed to date, He I $584 \AA$ data also exist and are undergoing detailed analysis.

\section{Summary}

As EUV instruments have become more sensitive, with higher spectral and spatial resolution than the early exploratory ones, newer insights are being obtained regarding planetary atmospheres and their surfaces. Already several questions raised by the Voyager mission have been addressed by the Astro-1 and EUVE missions. The Astro-2 mission and several sounding rocket experiments have obtained new data on several solar system objects. Results from these data have not been reported yet. We also note that neither $E U V E$ nor $H U T$ is ideal for solar system studies, since $E U V E$ was designed to either avoid detection or otherwise suppress many spectral features found in planetary atmospheres, and HUT lacked imaging capability. Progress in EUV instruments and availability of space flight opportunity will undoubtedly continue to increase our knowledge and understanding of the solar system.

We acknowledge the support of NASA grant NAG5-2260.

\section{REFERENCES}

Bertaux, J. -L., Blamont, J. E. Lepine, V. M, Kurt, V. G., Romanova, N. N. \& SMIRNov, A. S. 1981, Venera 11 and Venera 12 Observations of EUV Emissions from the Upper Atmosphere of Venus, Planet. Space Sci., 29, 149

BRoADFoot, A. L. 1977, Ultraviolet Spectrometer Experiment for the Voyager Mission, Space Sci. Rev., 21, 183

Broadfoot, A. L., ET Al. 1979, Extreme Ultraviolet Observations from Voyager 1 Encounter with Jupiter, Science, 204, 979

Broadfoot, A. L., ET AL. 1989, Ultraviolet Spectrometer Observations of Neptune and Triton, Science, 246, 1459 
Bush, B. C. \& Chakrabarti, S. 1995, Analysis of Lyman $\alpha$ and He I $584 \AA$ Airglow Measurements using a Spherical Radiative Transfer Model, J. Geophys. Res., in press

Chassefiere, E., Bertaux, J. -L., Kurt, V. G. \& Smirnov, A. S. 1986, Venus EUV Measurements of Helium at $58.4 \mathrm{~nm}$ from Venera 11 and Venera 12 and Implication of Outgassing History, Planet. Space Sci., 34, 585

Chassefiere, E., Dalaudier, F., \& Bertaux, J. L. 1988, Estimate of Interstellar Helium Parameters from Prognoz 6 and Voyager 1/2 EUV Resonance Glow Measurements Taking into Account a Possible Redshift in the Solar Line Profile, A\&A, 201, 113

Chiu, Y. T., Robinson, R. M., Swenson, G. R., Chakrabarti, S., \& Evans, D. S. 1986, Imaging the Outflow of Ionospheric Ions in the Magnetosphere, Nature, 322, 441

Clarke, J. T., Hudson, M. K., \& Yung, Y. L. 1987, The Excitation of Far Ultraviolet Electroglow Emissions on Uranus, Saturn and Jupiter, J. Geophys. Res., 92, 15,139

Cotton, D. M., Gladstone, G. R., Chakrabarti, S., \& Link, R. 1993, Sounding Rocket Observations of a Hot Atomic Oxygen Geocorona, J. Geophys. Res., 98, 21,651

Edwards, B. C., Priedhorsky, W. C. \& SMith, B. H. 1991, Expected Extreme Ultraviolet Spectrum of the Lunar Surface, Geophys. Res. L, 18, 2161

Feldman, P. D., \& Bagenal, F. 1991, EUV Planetary Astronomy, Extreme Ultraviolet Astronomy, R. F. Malina and S. Bowyer Eds, Pergamon, 252

Feldman, P. D., \& Morrison, D. 1991, The Apollo 17 Ultraviolet Spectrometer: Lunar Atmosphere Measurements Revisited, Geophys. Res. L., 18, 2105

FELDMAN, P. D. ET AL. 1991, Observations of Comet Levy 1990XX, with the Hopkins Ultraviolet Telescope, The ApJ, 379, L37

Feldman, P. D. ET AL. 1992, The Spectrum of Tropical Oxygen Nightglow Observed at $3 \AA$ Resolution with the Hopkins Ultraviolet Telescope, Geophys. Res. L., 19, 453

Feldman, P. D., McGrath, M. A., Moos, H. W., Durrance, S. T., Strobel, D. F., \& DAVIDSEN, A. F. 1993, The Spectrum of Jovian Dayglow Observed at $3 \AA$ with the Hopkins Ultraviolet Telescope, ApJ, March 93, 279

Gervais, F. 1991, Aluminum Oxide $\mathrm{Al}_{2} \mathrm{O}_{3}$, in Handbook of Optical Constants of Solids II, ed. E. D. Palik, New York: Academic Press, 761

Gladstone, G. R. 1994, Extreme Ultraviolet Observations of the Moon, Proceedings of the Tenth Thematic Conference on geologic Remote Sensing, I, 275, Environmental Research Institute of Michigan, Ann Arbor

Gladstone, G. R., Hall, D. T., \& Waite, J. H., JR. 1995, EUVE Observations of Jupiter During the Impact of Comet Shoemaker-Levy 9, Science, 268, 1595

Gladstone, G. R., McDonald, J. S., Boyd, W. T., \& Bowyer, S. 1994, EUVE Photometric Observations of the Moon, Geophys. Res. L., 21, 461

Green, J. C., Cash, W., Cook, T. A., \& Stern, S. A. 1991, The Spectrum of Comet Austin from 910 to $1180 \AA$, Science, 251, 408

HaLL, D. T., ET AL. 1994, Extreme Ultraviolet Explorer Satellite Observation of Jupiter's Io Plasm Torus, ApJ, 426, L51

Hall, D. T., ET AL. 1995, Io Torus EUV Emissions During the Comet Shoemaker-Levy/9 Impacts, Geophys. Res. L., submitted

Holberg, J. B. 1986, Far-ultraviolet Background Observations at High Galactic Latitude, II, Diffuse Emission, ApJ, 311, 969

HORD, C. W., ET AL. 1991, Galileo Ultraviolet Spectrometer Experiment: Initial Venus and Interplanetary medium Cruise Results, Science, 253, 1548

Jelinsky, P., VAllerga, J. V., \& Edelstein, J. 1995, First Spectral Observations of the Diffuse Background with the Extreme Ultraviolet Explorer, ApJ, 442, 653

Krasnopolsky, V. A., Bowyer, C. S., Chakrabarti, S., Gladstone, G. R., \& McDonALD, J. S. 1994, First measurement of Helium on Mars: Implication for the Problem of Radiogenic Gases on the Terrestrial Planets, Icarus, 109, 337

MEIER, R. R. 1991, Ultraviolet Spectroscopy and remote Sensing of the Upper Atmosphere, 
Space Sci. Rev., 58, 1

Moos, H. W., ET AL. 1991, Determination of Ionic Abundances in the Io Torus Using the Hopkins Ultraviolet Telescope, ApJ, 382, L105

Ogawa, H., PhIllips, E., \& Judgle, D. L. 1984, Line Width of the Solar EUV He I Resonance Emissions at 584 and $537 \AA$, J. Geophys. Res., 89, 7537

PhILIPP, H. R. 1985, Silicon Dioxide $\mathrm{SiO}_{2}$, Glass, in Handbook of Optical Constants of Solids, ed. E. D. Palik, New York: Academic Press, 749

PARESCE, F., Fahr, H, \& LAY, G. 1981, A Search for Interplanetary He II $304 \AA$ emission, J. Geophys. Res., 86, 10038

Schmitt, J. H. M. M., Snowden, S. L., Aschenbach, B., Hasinger, G., Pfeffermann, E., Predehl, P., \& TrüMPen, J. 1991, A Soft X-Ray Image of the Moon, Nature, 349, 583

Slanger, T. G. 1991, The Spectrum of Comet Austin, Science, 253, 452

Stern, S. A., Green, J. C., CASH, W., \& CoOK, T. A. 1992, Helium and Argon Abundance Constraints and the Thermal Evolution of Comet Austin 1989c1, Icarus, 95, 157

Waite, J. H., ET AL. 1983, Superthermal Electron Processes in the Upper Atmosphere of Uranus: Aurora and Electroglow, J. Geophys. Res., 88, 6143

Wu, H. H., \& Broadfoot, A. L. 1977, The Extreme Ultraviolet Albedos of the Planet Mercury and of the Moon, J. Geophys. Res., 82, 759

Yelle, R. V., McConnell, J. C., Sandel, B. L., \& Broadfoot, A. L. 1987, The Dependence of Electroglow on the Solar Flux, J. Geophys. Res., 92, 15110 